\title{
Biomasa forestal, carbono fijado y almacenado en sistema bosque y sistema silvopastoril en siete fincas del municipio de Mulukukú, RACN, 2018
}

\section{Forest biomass, carbon fixed and stored in forest system and silvopastoral system in seven farms of the municipality of Mulukukú, RACN, 2018}

\author{
Nadieska Isabel Sánchez Rodas ${ }^{1}$, Norwuin Ali Irías Gutiérrez ${ }^{1}$, Claudio Calero González ${ }^{2}$, Edwin A. Alonzo Serrano ${ }^{3}$ \\ ${ }^{1}$ Ingeniero Forestal graduado de la Universidad Nacional Agraria, nadieskasanchez90@gmail.com / norwinali93@gmail.com \\ ${ }^{2}$ Profesor-Investigador Facultad de Recursos Naturales y del Ambiente (q.e.p.d) \\ ${ }^{3}$ MSc. Profesor Investigador Facultad de Recursos Naturales y del Ambiente, ORCID: https://orcid.org/0000-0002-9686-4772 \\ Edwin.Alonzo@ci.una.edu.ni
}

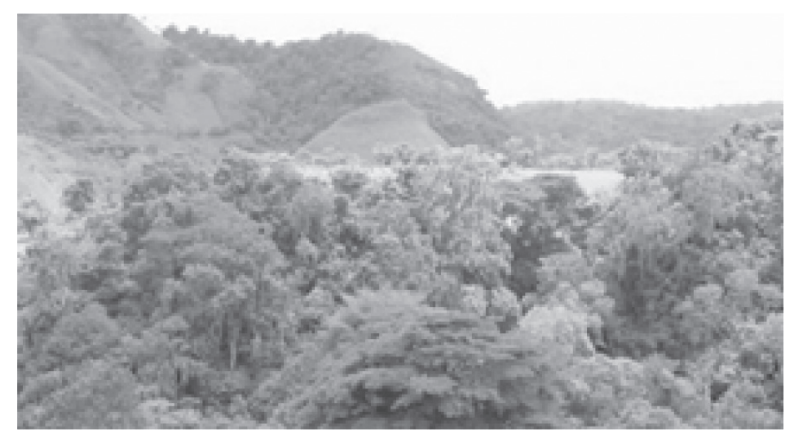

\section{RESUMEN}

La información básica de la capacidad de los sistemas bosques y silvopastoril en la fijación y almacenamiento de carbono en la biomasa aérea servirá de guía a los productores para conservar y manejar sus áreas. El presente estudio se realizó en siete fincas de productores del municipio de Mulukukú, Siuna, Región Autónoma del Caribe Norte (RACN), con el objetivo de determinar el carbono fijado y almacenado en la biomasa aérea presente en el componente arbóreo en sistemas bosques y silvopastoriles utilizando métodos no destructivos, identificación de especies más representativas y la determinación de la biomasa forestal, carbono fijado y almacenado. Se realizó un diseño de inventario en parcelas de Muestreo Permanentes (PMP); en el sistema bosque tres parcelas de 50 × 50 $\mathrm{m}$, dos en la finca La Ceiba y uno en la finca Regalo de Dios. En el sistema silvopastoril parcelas de 10 × $25 \mathrm{~m}$, dos por cada finca para un total de 14 parcelas. La densidad de la madera usada de $0.5 \mathrm{~g} \mathrm{~cm}^{3}$ convertido a $\mathrm{kg} \mathrm{m}^{3}$ y un factor de expansión de 1.20 para biomasa aérea (ramas, hojas) establecidas por FAO (1997 y 1998). Para el carbono almacenado se utilizó la fracción de 0.5 asumiendo que el $50 \%$ de biomasa es carbono. El $\mathrm{CO}_{2}$ fijado se calculó en base al carbono de la madera multiplicado por 3.67 que sale directamente del peso atómico del $\mathrm{CO}_{2}$. Como resultado de la investigación; el sistema bosque la riqueza de especies se contabiliza en 238 individuos, perteneciente a 40 especies, 38 géneros y 28 familias. Sistemas silvopastoril 136 individuos, 24 especies, 24 géneros y 23 familias. El promedio de biomasa seca para el sistema bosque es

\begin{abstract}
Basic information on the capacity of forest and silvopastoral systems to fix and store carbon in aerial biomass will guide producers to conserve and manage their areas. The present study was carried out in seven producer farms in the municipality of Mulukukú, Siuna, Autonomous Region of the Northern Caribbean (RACN), with the objective of determining the carbon fixed and stored in the aerial biomass present in the tree component in forest and silvopastoral systems using non-destructive methods, identification of the most representative species and the determination of forest biomass, fixed and stored carbon. An inventory design was carried out in Permanent Sampling plots (PMP); in the forest system three plots of 50 x $50 \mathrm{~m}$, two on the La Ceiba farm and one on the Regalo de Dios farm. In the silvopastoral system, plots of $10 \times 25 \mathrm{~m}$, two for each farm for a total of 14 plots. The density of the used wood of $0.5 \mathrm{~g} \mathrm{~cm}^{3}$ converted to $\mathrm{kg} \mathrm{m}^{3}$ and an expansion factor of 1.20 for aerial biomass (branches, leaves) established by FAO (1997 and 1998). For the stored carbon, the fraction of 0.5 was used assuming that $50 \%$ of biomass is carbon. The fixed $\mathrm{CO}_{2}$ was calculated based on the carbon of the wood multiplied by 3.67 that comes directly from the atomic weight of the $\mathrm{CO}_{2}$. As a result of the investigation; the forest system species richness is recorded in 238 individuals, belonging to 40 species, 38 genera and 28 families. Silvopastoral systems 136 individuals, 24 species, 24 genera and 23 families. The average dry biomass for the forest system is $85.6269 \mathrm{t} \mathrm{ha}^{-1}$, $157.12 \mathrm{t} \mathrm{ha}^{-1} \mathrm{CO}_{2}$ and $42.80 \mathrm{t} \mathrm{ha}^{-1}$ carbon, silvopastoral system
\end{abstract}

Recibido: 6 de septiembre del 2019 Aceptado: 20 de abril del 2020

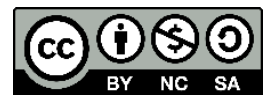

C Copyright 2020. Universidad Nacional Agraria (UNA).
Los artículos de la revista La Calera de la Universidad Nacional Agraria, Nicaragua, se comparten bajo términos de la licencia Creative Commons: Reconocimiento, No Comercial, Compartir Igual. Las autorizaciones adicionales a las aquí delimitadas se pueden obtener en el correo edgardo.jimenez@ci.una.edu.ni 
85.62 $69 \mathrm{t} \mathrm{ha}^{-1}, 157.12 \mathrm{t} \mathrm{ha}^{-1} \mathrm{CO}_{2}$ y $42.80 \mathrm{t} \mathrm{ha}^{-1}$ carbono, sistema silvopastoril $20.05 \mathrm{t} \mathrm{ha}^{-1}, 36.79 \mathrm{t} \mathrm{ha}^{-1} \mathrm{CO}_{2}$ y $42.80 \mathrm{t} \mathrm{ha}^{-1}$ carbono. El sistema bosque registra mayor valor en promedio de biomasa seca, dióxido de carbono fijado y almacenado por hectárea, superiores a los encontrados en sistemas silvopastoriles ya que las fincas presentan diferencias en cantidad de árboles por hectáreas, y el manejo tecnificado por los dueños.

Palabras clave: parcela de muestreo, biomasa, carbono almacenado. $\mathrm{CO}_{2}$ fijado.
$20.05 \mathrm{t} \mathrm{ha}^{-1}, 36.79 \mathrm{t} \mathrm{ha}^{-1} \mathrm{CO}_{2}$ and $42.80 \mathrm{t} \mathrm{ha}^{-1}$ carbon. The forest system registers a higher average value of dry biomass, fixed and stored carbon dioxide per hectare, higher than those found in silvopastoral systems since the farms show differences in the number of trees per hectare, and the technified management by the owners.

Keywords: Sampling Plot, biomass, stored carbon, $\mathrm{CO}_{2}$ fixed.

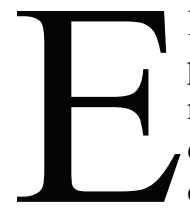

1 cambio climático representa una seria amenaza para las sociedades centroamericanas por sus múltiples impactos previstos en la población y en los sectores productivos. Se reconoce que el calentamiento global es producto de una serie de acciones del hombre, dentro de las cuales se destaca a nivel Centroamericano la deforestación y cambio de uso del suelo, cuyas consecuencias han sido enormes por pérdidas en bienes materiales, biodiversidad y de la misma vida humana (Ramírez, Ordaz, Mora, Acosta y Cerna, 2010).

Es por esta razón que los bosques como sistema natural complejo y sumideros de carbono, contribuyen a mitigar el cambio climático global al almacenar grandes cantidades de carbono en la vegetación y en el suelo, e intercambiar carbono con la atmósfera liberando oxígeno a través del proceso fotosintético y la respiración. No se deja de reconocer que, en el caso de alteraciones en los bosques, los mismos se convierten en fuentes de carbono liberando $\mathrm{CO}_{2}$ hacia la atmósfera (Gasparri et al., 2004).

Los sistemas silvopastoriles, al incluir el componente arbóreo, pueden remover cantidades significativas de carbono de la atmósfera al incorporarlo a su biomasa, además de otros beneficios productivos y ambientales. Estos sistemas se pueden constituir como importantes sumideros de carbono en altas proporciones debido a que se concentra carbono en los diferentes componentes tanto de la especie arbórea como la pastura en asocio (Ordoñez, Muñoz, Ballesteros, Cabrera y Mosquera, 2007).

Estos estudios son importantes para Nicaragua, ya que actualmente el país posee el $32.87 \%$ de su territorio con bosques protegidos bajo la categoría de parques nacionales; el bosque latifoliado está constituido por 1233988 ha en bosque primario y 135399 ha de bosque secundario. Posee además aproximadamente 73679 ha de plantaciones forestales, con diversas especies [Instituto Nacional Forestal (INAFOR, 2009)].

El objetivo de esta investigación es aportar información básica sobre la capacidad que tienen los sistemas bosques y silvopastoril en la fijación y almacenamiento de carbono presente en la biomasa aérea en sitios seleccionados del municipio de Mulukukú, Región Autónoma del Caribe Norte de Nicaragua, es importante resaltar que el presente estudio mediante los resultados obtenidos proporcionará información y servirá como guía para los productores de fincas para conservar y manejar sus áreas de bosque $\mathrm{y}$ sistemas silvopastoril, así como también será de gran utilidad para el proyecto de la Comisión Nacional Ganadera de Nicaragua (CONAGAN) y la Universidad Nacional Agraria (UNA). Estos resultados de biomasa y carbono servirán de referencia para otros estudios en la región, o bien para estudios más locales, o más específicos y tomen como base esta información.

\section{MATERIALES Y MÉTODOS}

En la Figura 1, se presenta el Municipio de Mulukukú, se sitúa al suroeste de Puerto Cabezas pertenece a la Región Autónoma del Caribe Norte (RACN), el municipio es nuevo debido a que fue fundado en el año 2005. Se encuentra a $284 \mathrm{~km}$ de la ciudad de Managua. Limita Al Norte: con el Municipio de Siuna. Al Sur: Con los municipios de Río Blanco y Paiwas, Al Este: Con los municipios de Prinzapolka y Cruz de Rio Grande y al Oeste: Con el municipio de Waslala [Instituto Nicaragüense de Estudios Territoriales (INETER, 2009)].

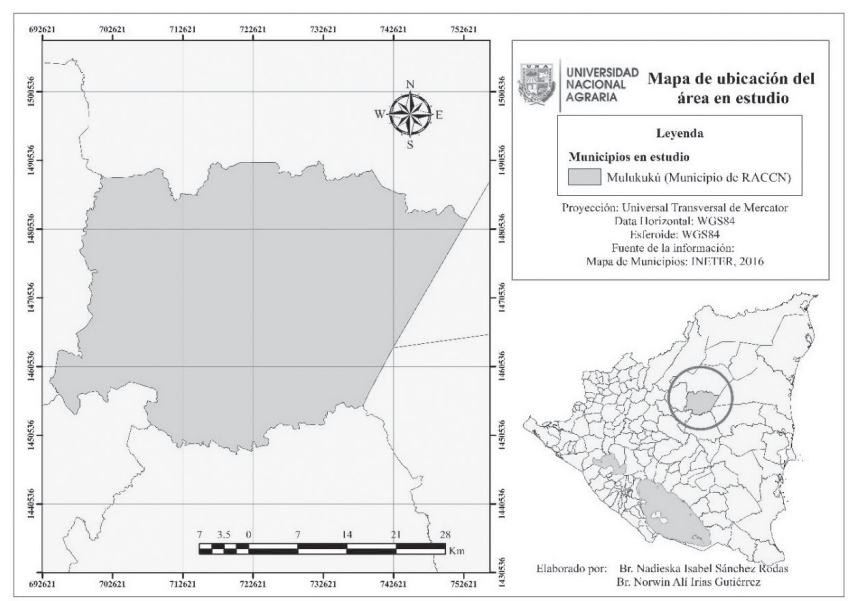

Figura 1. Ubicación de Mulukukú, Región Autónoma del Caribe Norte. 
En la presente investigación se estudiaron sistema bosque y silvopastoril con el objetivo de cuantificar la biomasa aérea y carbono almacenado en el componente arbóreo mediante el método no destructivo de siete fincas seleccionadas del municipio de Mulukukú Región Autónoma del Caribe Norte (RACN).

Etapa I. Planificación del estudio. Esta primera etapa estuvo centrada principalmente para establecer acuerdos y definir aspectos técnicos y logísticos. Estos acuerdos fueron tomados entre las partes CONAGAN-Productores - UNA.

Las fincas evaluadas en el presente estudio fueron seleccionadas por el proyecto ganadería sostenible de Nicaragua (CONAGAN), una vez en el sitio para la visita de cada finca y levantamiento de información de campo, nos apoyamos por el técnico designado por CONAGAN-Carlos Hogson, el cual nos trasladaba a las fincas que se habían seleccionado y según sus conocimientos de la localidad nos informó que las siete fincas se encontraban dentro del municipio de Mulukukú, hacemos mención de esto porque al momento de incorporar los datos de georreferenciación de las parcelas y utilizar la cartografía para elaborar el mapa de ubicación y establecimiento de cada parcela, resulta que seis fincas están ubicadas en el municipio de Mulukukú y una finca (Regalo de Dios) se sitúa en la comunidad Guasayamba municipio de Paiwas, esta finca no se refleja en el mapa de Mulukukú por lo antes mencionado, pero si fue evaluada y valorada en los resultados obtenidos en nuestro estudio.

En el Cuadro 1, se presenta el listado de las siete fincas del municipio de Mulukukú (RACN) y sus propietarios, los cuales estaban de acuerdo en que se desarrollara este trabajo.

Cuadro 1. Fincas seleccionadas en el municipio de Mulukukú, Siuna

\begin{tabular}{lll}
\hline \multicolumn{1}{c}{ Nombre de fincas } & \multicolumn{1}{c}{ Comunidad } & \multicolumn{1}{c}{ Productores } \\
\hline La Ceiba & El tigre & Andrés Rivas López \\
Las Marías & Lisague & Feliciano Lira \\
Santa Emilia & & Rosa Emilia Lira \\
& Lisague & Montenegro \\
Buena Vista & Lisague & Ramón Gutiérrez Zamora \\
Buenos Aires & Lisague & Sidar Noel Hurtado Alfaro \\
El Cacao & Lisague & Lucas Lira Montenegro \\
Regalo de Dios & Guasayamba & Wilfredo Zeledón \\
\hline
\end{tabular}

\section{Etapa II: Proceso de levantamiento de información}

Diseño del inventario por tipo de sistema. Se estableció un diseño de inventario en parcelas de muestreo permanente (PMP) por ser más eficientes y para realizar mediciones futuras, las cuales se establecieron en las fincas seleccionadas en sistemas bosques y silvopastoril (Figura 2).
Forma y tamaño de cada parcela. En el sistema bosque la parcela utilizada fue de forma cuadrada de $50 * 50 \mathrm{~m}^{2}(0.25$ ha) dentro de esta parcela se midieron todos aquellos árboles que cumplían con el diámetro establecido $\geq 10 \mathrm{~cm}$ DAP. En áreas de sistemas Silvopastoril la parcela utilizada fue de forma rectangular de $10 * 25 \mathrm{~m}^{2}(0.025 \mathrm{ha})$, donde se midieron todos aquellos árboles que cumplían con el diámetro $\geq 10 \mathrm{~cm}$ de DAP, más no el componente pastoril (Figura 2).

Establecimiento de parcelas. En el sistema bosque se establecieron dos parcelas distanciadas $200 \mathrm{~m}$ en la finca La Ceiba y en la finca Regalo de Dios se estableció una parcela debido a que el bosque era pequeño. Para el establecimiento de las parcelas de muestreo permanentes (PMP) se contó con el criterio de que en el área se encontrara cubierta de masa forestal no importando que esta fuera rala o densa. Se midieron las parcelas equivalentes a $0.25 \mathrm{ha}$, tomando como punto de partida de cada parcela un árbol de referencia, luego se procedió a marcar los puntos de cada parcela utilizando un primer azimut como punto A de $290^{\circ}$ girado hacia el Nor-oeste, un punto B de $200^{\circ}$ Sur-Oeste, punto C de $110^{\circ}$ Sur-Este y un último punto $\mathrm{D}$ de $20^{\circ}$ Nor-Este. Aplicando este método a las tres parcelas que se establecieron en áreas de bosque. En sistemas silvopastoril para cada finca se establecieron dos parcelas de muestreo para un total de 14 parcelas en siete fincas dentro del municipio, se contó con el criterio de que en el área donde se establecieran las parcelas se encontraran árboles dispersos. Procediendo a marcar cada punto de la parcela utilizando como azimut punto A de $310^{\circ}$ girando hacia la izquierda Nor-Oeste, un segundo punto $\mathrm{B}$ de $40^{\circ}$ Nor-Este, punto $\mathrm{C}$ de $130^{\circ}$ Sur-Este y punto D de $220^{\circ}$ Sur-Oeste. Realizando este método a todas las parcelas establecidas en sistemas silvopastoriles.

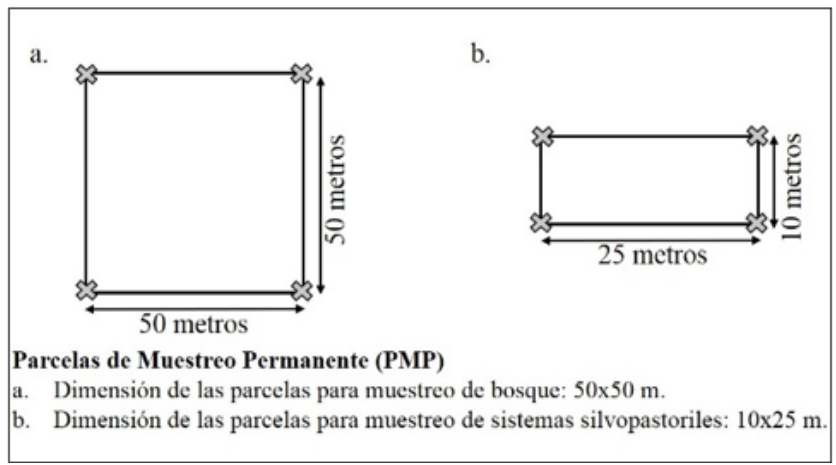

Figura 2. Diseño metodológico en parcelas de muestreo permanentes (PMP) en sistema bosque y silvopastoril en fincas seleccionadas del municipio de Mulukukú (RACN) 2018. 
Cálculos utilizando las variables dasométricas

Distribución diamétrica: Determinación del número de clases diamétricas $\left(\mathrm{N}^{\circ} \mathrm{CD}\right)$ :

$\mathrm{N}^{\circ} \mathrm{CD}=\left(\mathrm{D}_{\mathrm{M}}-\mathrm{D}_{\mathrm{m}}\right) / \mathrm{AC}$

Donde:

$\mathrm{N}^{\circ} \mathrm{CD}$ : Número de clases diamétrica

DM: Diámetro mayor de la base de datos

Dm: Diámetro menor de la base de datos

AC: Amplitud de clase según los intervalos de tamaño adecuado. La amplitud de clases establecida para este caso es de $10 \mathrm{~cm}(10-19.99 \mathrm{~cm})$

Cálculo del área basal: Para calcular el área basal, según (Ugalde, 1981), se puede determinar de la siguiente manera:

$\mathrm{AB}\left(\mathrm{m}^{2}\right)=\pi / 4 * \mathrm{D}^{2}$

Dónde:

$\mathrm{AB}$ : Área basal $\left(\mathrm{m}^{2}\right)$

$\pi / 4$ : constante 0.7854

DN: Diámetro normal medido a los $1.30 \mathrm{~m}$ sobre la superficie del suelo.

Cálculo del volumen: Para calcular el de Volumen $\left(\mathrm{m}^{3}\right)$, según (Ugalde, 1981), se puede determinar de la siguiente manera:

$\operatorname{Vol}\left(\mathrm{m}^{3}\right)=\mathrm{AB} * \mathrm{Ht} * \mathrm{Ff}$

Dónde:

Vol. volumen $\left(\mathrm{m}^{3}\right)$

$\mathrm{AB}$ : área basal calculada en $\mathrm{m}^{2}$

Ht: Altura total en (m)

Ff: Factor de forma 0.5 para especies de bosque latifoliados (Mejía, 2013).

Cálculo de la biomasa forestal: Para calcular la biomasa forestal (ton), según (Brown, 1997), se puede determinar con la siguiente formula:

\section{$\mathrm{Bf}=\mathrm{V} * \mathrm{GE} * \mathrm{FEBa}$}

Dónde:

Bf: Biomasa forestal (ton)

$\mathrm{V}$ : Volumen total calculado en $\mathrm{m}^{3}$

GE: Densidad de la madera $0.5 \mathrm{~g} \mathrm{~cm}^{3}$ según (Brown, 1997).

FEBa: Factor de expansión de la biomasa aérea (ramas, hojas) [es de 1.20, según la Organización de las Naciones Unidas para la Alimentación y la Agricultura (FAO, 1998)].
Conversión de $\mathrm{g} / \mathrm{cm}^{3}$ a kg/m

$0.5 \mathrm{~g}$
$\mathrm{~cm}^{3}$$\frac{\mathrm{kg}}{\mathrm{m}^{3}} \quad \begin{aligned} & 1 \mathrm{~kg}=1000 \mathrm{~g} \\ & 1 \mathrm{~m}=100 \mathrm{~cm} \\ & 1 \mathrm{~m} 3=1000000 \mathrm{~cm}^{3}\end{aligned}$

$\frac{0.5 \mathrm{~g}}{\mathrm{em}^{3}}-\frac{1 \mathrm{~kg}}{1000 \mathrm{~g}}$
$\frac{0.5 * 1 \mathrm{~kg} * 1000000}{1000 * 1 \mathrm{~m}^{3}}=\frac{1000000 \mathrm{~km}^{2}}{1 \mathrm{~m}^{3}}$
$\frac{500000 \mathrm{~kg}}{1000 \mathrm{~m}^{3}}=500 \mathrm{~kg} \mathrm{~m}^{3}$

Cálculo del carbono almacenado: Para calcular el carbono almacenado (ton), según (Rügnitz, Chacon y Porro, 2009), se puede determinar con la siguiente formula:

$\mathrm{C}=\mathrm{Bf} * \mathrm{Fc}$

Dónde:

C: Carbono almacenado (ton)

Bf: Biomasa forestal (ton)

Fc: Factor de carbono (0.5) (Jenkins et al., 2006)

Cálculo del dióxido de carbono fijado $\left(\mathrm{CO}_{2}\right)$ : Para calcular el carbono fijado (ton), según (Rügnitz et al., 2009), se puede determinar con la siguiente formula:

$\mathrm{CO}_{2}=\mathrm{C} * 3.67$

Dónde

$\mathrm{CO}_{2}$ : Carbono fijado (ton)

$\mathrm{C}$ : Carbono almacenado calculado en (ton)

Constante $=3.67\left(\right.$ Peso atómico del $\mathrm{CO}_{2}$ )

Relación de Carbono - Dióxido de carbono, considerando el peso molecular del $\mathrm{CO}_{2}$ :

$\mathrm{C}=12$ (peso atómico)

$\mathrm{O}=16$ (peso atómico)

Ecuación química

$\mathrm{CO}_{2}=\mathrm{C}+2 * \mathrm{O}$ (Walker et al., 2011)

$\mathrm{CO}_{2}=12+2(16)=44$ (peso molecular)

$\mathrm{R}=\mathrm{CO}_{2} / \mathrm{C}$ (Relación de peso, $\mathrm{CO}_{2}$ respecto al carbono)

$\mathrm{R}=44 / 12$ (Walker et al., 2011)

$\mathrm{R}=3.67$ (para obtener una unidad de carbono se requieren 3.67 unidades de $\mathrm{CO}_{2}$ ). 
Sistema bosque

\section{RESULTADOS Y DISCUSIÓN}

Comportamiento de la biomasa forestal total por especie en $t$ ha $^{-1}$ encontrada en la finca La Ceiba en el sistema bosque. En la finca La Ceiba el mayor valor de biomasa forestal por hectárea lo presentó el roble macuelizo (Tabebuia rosea) con $18.61 \mathrm{t} \mathrm{ha}^{-1}$, seguido de la especie carao (Casia grandis) con $14.18 \mathrm{t} \mathrm{ha}^{-1}$, y finalmente la ceiba (Ceiba pentandra) $13.04 \mathrm{t} \mathrm{ha}^{-1}$, otras especies $55.94 \mathrm{t} \mathrm{ha}^{-}$ ${ }^{1}$, encontrando un total de biomasa forestal para todas las especies de $101.77 \mathrm{t} \mathrm{ha}^{-1}$ (Figura 3). Se observó que la mayor concentración de biomasa seca se dio en esta finca por ser la que mayor cobertura de bosque posee, debido a que las especies presentaron mayor densidad de árboles y mayores dimensiones en diámetro y altura.

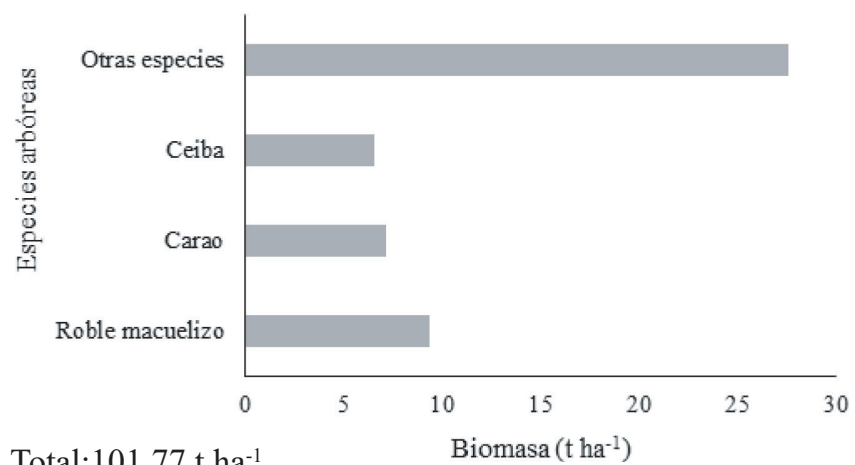

Total:101.77 $\mathrm{t} \mathrm{ha}^{-1}$

Biomasa $\left(\mathrm{t} \mathrm{ha}^{-1}\right)$

Figura 3. Biomasa forestal por especie $\left(\mathrm{t} \mathrm{ha}^{-1}\right)$ finca La Ceiba, Mulukukú, 2018.

Análisis de la fijación de dióxido de carbono total $\left(\mathrm{CO}_{2}\right)$ encontrado en la finca La Ceiba. Las especies que presentan mayor fijación de dióxido de carbono $\left(\mathrm{CO}_{2}\right)$ son: Roble macuelizo (Tabebuia rosea) con $34.15 \mathrm{t} \mathrm{ha}^{-1}$, en segundo lugar, la especie carao (Casia grandis) con $26.02 \mathrm{t} \mathrm{ha}^{-1}$, en tercero la ceiba (Ceiba pentandra) con $23.93 \mathrm{t} \mathrm{ha}^{-1}$, otras especies $102.65 \mathrm{t} \mathrm{ha}^{-1}$, registrando un total de carbono fijado para todas las especies de $186.75 \mathrm{t} \mathrm{ha}^{-1}$ (Figura 4). Se puede notar que la finca La Ceiba es la que más está aportando a la fijación de carbono $\left(\mathrm{CO}_{2}\right)$, debido a que su composición de especies arbórea presenta más carbono almacenado en su biomasa acumulada en árboles con mayores dimensiones en diámetro, en comparación a la composición florística de la finca Regalo de Dios del mismo estudio.

Carbono almacenado en la finca La Ceiba. Las especies que presentan mayor cantidad de carbono almacenado son: Roble macuelizo (Tabebuia rosea) con $9.3 \mathrm{t} \mathrm{ha}^{-1}$, seguido de la especie carao (Casia grandis) con $7.09 \mathrm{t} \mathrm{ha}^{-1}$, en tercer lugar, la ceiba (Ceiba pentandra) con $6.52 \mathrm{t} \mathrm{ha}^{-1}$, otras especies $27.57 \mathrm{t} \mathrm{ha}^{-1}$, encontrando un total de carbono almacenado para todas las especies de $50.88 \mathrm{t} \mathrm{ha}^{-1}$ (Figura 5). Se observó que en la finca La Ceiba el carbono almacenado en relación a la composición florística presento mayores resultados debido a que se encontró mayor riqueza de especies, y mayor densidad de árboles.

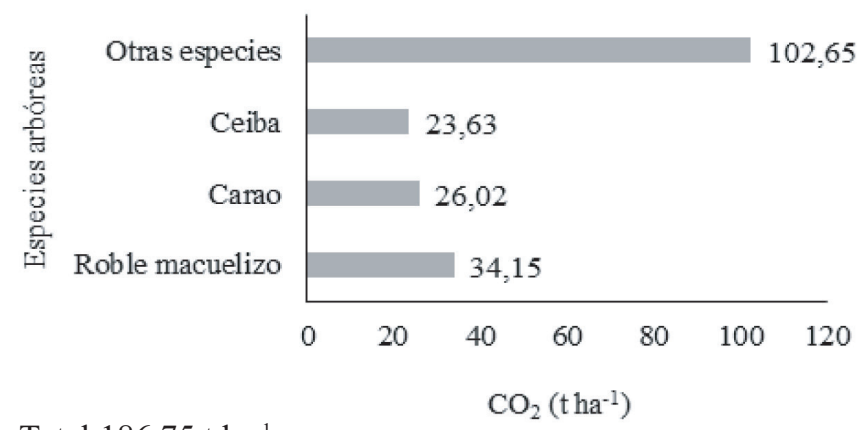

Total:186.75 $\mathrm{t} \mathrm{ha}^{-1}$

Figura 4. Carbono fijado $\mathrm{CO}_{2}$ por especie $\left(\mathrm{t} \mathrm{ha}^{-1}\right)$ finca La Ceiba, Mulukukú, 2018.

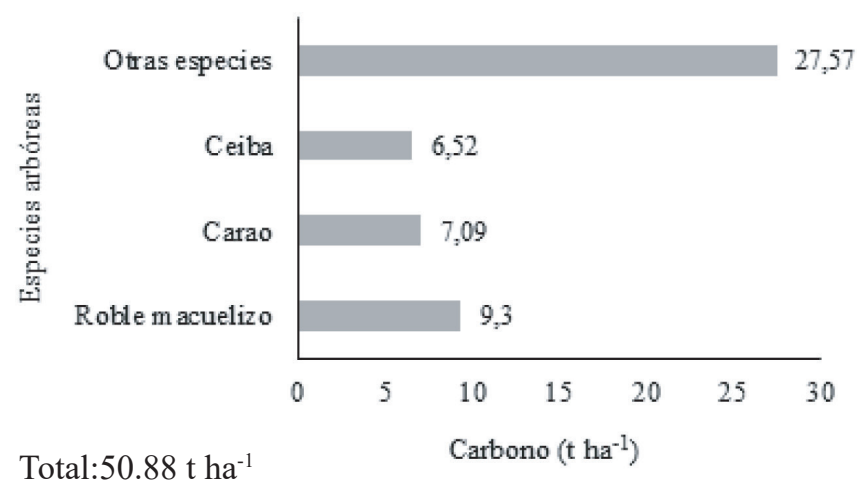

Figura 5. Carbono almacenado por especie $\left(\mathrm{t} \mathrm{ha}^{-1}\right)$ en la finca $\mathrm{La}$ Ceiba, Mulukukú, 2018.

Comportamiento de la biomasa seca total por especie en $\mathbf{t ~ h a}^{-1}$ encontrada en la finca Regalo de Dios. En la finca Regalo de Dios el mayor valor de biomasa forestal por hectárea lo presenta el chinche (Zanthoxylum fagara) con $21.90 \mathrm{t} \mathrm{ha}^{-1}$, en segundo lugar, la especie nancitón (Hyeronima alchorneoides) con $11.66 \mathrm{t} \mathrm{ha}^{-1} \mathrm{y}$ en tercero el roble macuelizo (Tabebuia rosea) con $11.63 \mathrm{t} \mathrm{ha}^{-1}$, otras especies $24.28 \mathrm{tha}^{-1}$, registrando un total de biomasa forestal para todas las especies de $69.47 \mathrm{t} \mathrm{ha}^{-1}$ (Figura 6). Se pudo notar que esta finca presento una cobertura de bosque ralo y las especies presentaron menores dimensiones en diámetro y altura y por lo tanto el contenido de biomasa fue bajo en comparación a la finca La Ceiba del mismo estudio.

Análisis de la fijación de dióxido de carbono total $\left(\mathrm{CO}_{2}\right)$ encontrado en la finca Regalo de Dios. La especie con mayor fijación de dióxido de carbono es: Chinche (Zanthoxylum fagara) con $40.19 \mathrm{t} \mathrm{ha}^{-1}$, seguido de la especie nancitón (Hyeronima alchorneoides) con $21.39 \mathrm{t} \mathrm{ha}^{-1}$, y 


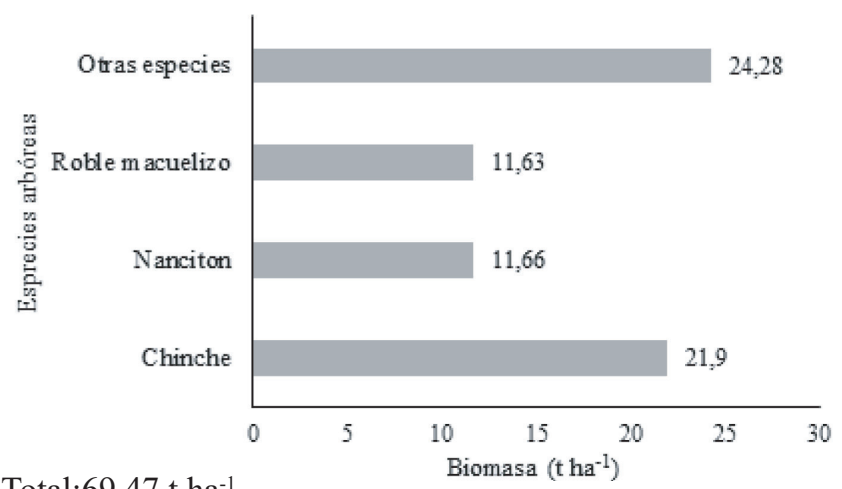

Total: $69.47 \mathrm{t} \mathrm{ha}^{-1}$

Figura 6. Biomasa forestal por especie $\left(\mathrm{t} \mathrm{ha}^{-1}\right)$ en la finca Regalo de Dios, Mulukukú, 2018.

finalmente roble macuelizo (Tabebuia rosea) con $21.34 \mathrm{tha}$ ${ }^{1}$, otras especies $44.57 \mathrm{t} \mathrm{ha}^{-1}$. El total de carbono fijado para todas las especies es de $127.49 \mathrm{t} \mathrm{ha}^{-1}$ (Figura 7). Se notó que la finca Regalo de Dios aporto en menor cantidad a la fijación de carbono en comparación a la finca La Ceiba del mismo estudio, debido a las pocas especies encontradas en este sitio y a los bajos contenidos de carbono en la biomasa de árboles que en su mayoría se encontraron en diámetros menores.

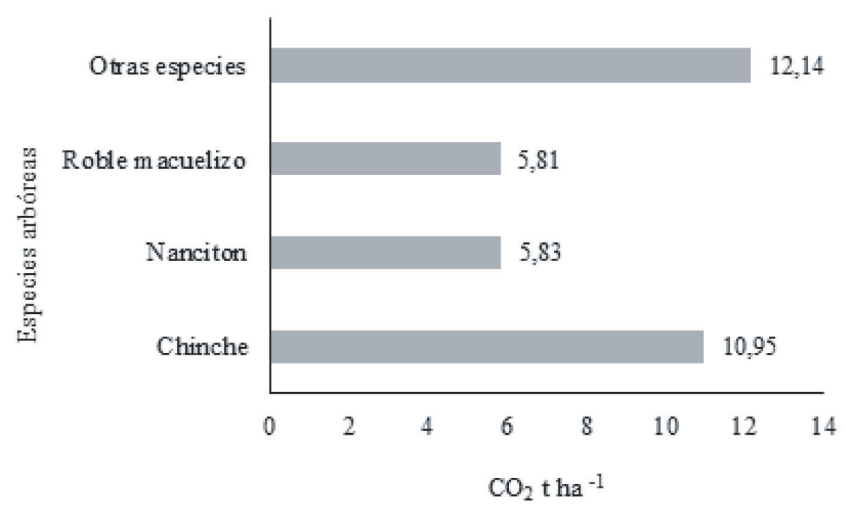

Total:127.49 $\mathrm{CO}_{2} \mathrm{t} \mathrm{ha}^{-1}$

Figura 7. Carbono Fijado $\mathrm{CO}_{2}$ por especie $\left(\mathrm{t} \mathrm{ha}^{-1}\right)$ en la finca Regalo de Dios, Mulukukú, 2018.

Carbono almacenado en la finca Regalo de Dios. La especie que presenta la mayor cantidad de carbono almacenado es: Chinche (Zanthoxylum fagara) con $10.95 \mathrm{t} \mathrm{ha}^{-1}$, seguido del nancitón (Hyeronima alchorneoides) con $5.83 \mathrm{t} \mathrm{ha}^{-1}$ y en tercer lugar el roble macuelizo (Tabebuia rosea) con $5.81 \mathrm{t}$ $\mathrm{ha}^{-1}$, otras especies $12.14 \mathrm{tha}^{-1}$. Se registró un total de carbono almacenado para todas las especies de $34.73 \mathrm{t} \mathrm{ha}^{-1}$ (Figura 8). El carbono almacenado en la finca Regalo de Dios fue menor, debido que la composición florística presento menor riqueza de especies, y la mayor parte de la biomasa se encontró en árboles con diámetros menores.

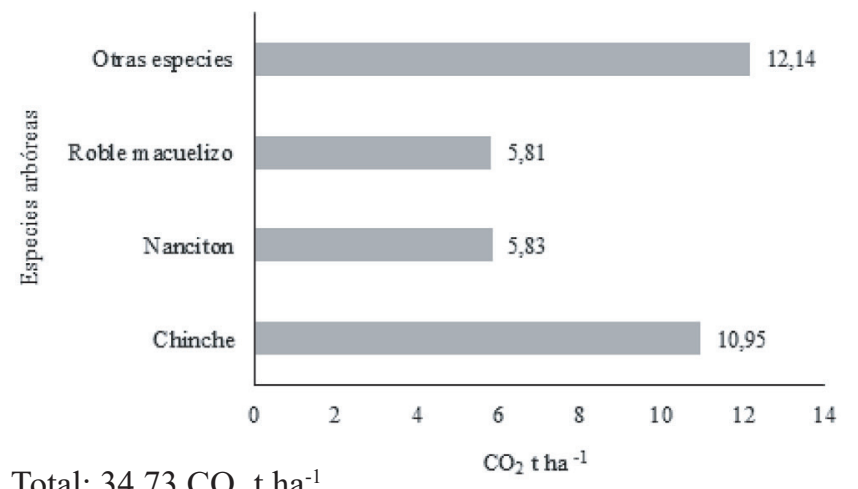

Total: $34.73 \mathrm{CO}_{2} \mathrm{t} \mathrm{ha}^{-1}$

Figura 8. Carbono almacenado por especie $\left(\mathrm{t} \mathrm{ha}^{-1}\right)$ en la finca Regalo de Dios, Mulukukú, 2018.

\section{Sistemas silvopastoriles}

Comportamiento de la biomasa forestal total en sistemas silvopastoriles. En las siete fincas en estudio el mayor valor de biomasa forestal por hectárea lo presenta el Roble macuelizo (Tabebuia rosea) con $74.93 \mathrm{t} \mathrm{ha}^{-1}$, seguido de la especie indio desnudo (Bursera simarouba) con $15.12 \mathrm{t} \mathrm{ha}^{-1}$ y finalmente la especie coyote (Platymiscium pleiostachyum) con $13.04 \mathrm{t} \mathrm{ha}^{-1}$, otras especies $37.25 \mathrm{t} \mathrm{ha}^{-1}$, para un total de todas las especies de $140.36 \mathrm{t} \mathrm{ha}^{-1}$ (Figura 9). Se puede notar que los resultados de biomasa forestal encontrado en sistema silvopastoril en relación con la composición de especies, se debe que los árboles presentaron altas dimensiones en diámetro y altura, a pesar que la cobertura arbórea en estos sistemas no se les da un manejo técnico, las especies presentan alta cantidad de árboles/ha y están aportando buena acumulación en totales de biomasa forestal, en comparación a los valores obtenidos en el sistema bosque del mismo estudio.

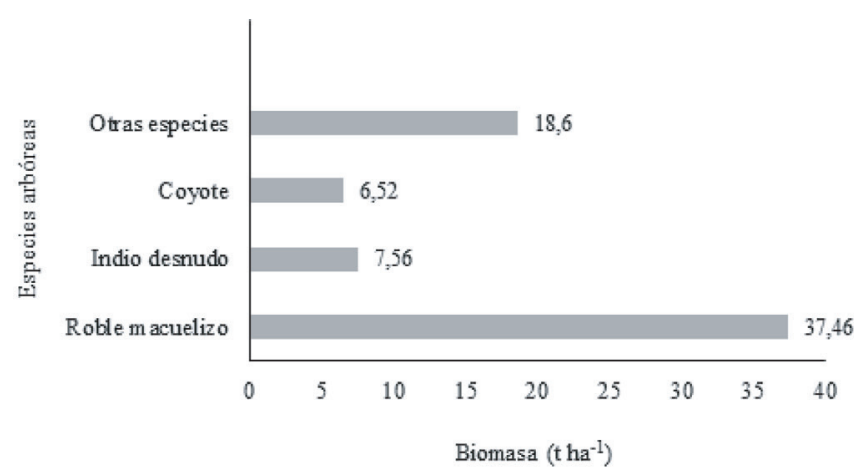

Total:140.36 $\mathrm{tha}^{-1}$

Figura 9. Biomasa forestal por especie $\left(\mathrm{tha}^{-1}\right)$ en siete fincas en los sistemas Silvopastoriles, Mulukukú, 2018.

Fijación de dióxido de carbono $\left(\mathrm{CO}_{2}\right)$ total en sistemas silvopastoriles. Las especies que presentan mayor cantidad de carbono fijado son: Roble macuelizo (Tabebuia rosea) 
con $137.50 \mathrm{t} \mathrm{CO}_{2} \mathrm{ha}^{-1}$, seguido de la especie Indio desnudo (Bursera simarouba) con $27.74 \mathrm{t} \mathrm{CO}_{2} \mathrm{ha}^{-1}$ y finalmente la especie coyote (Platymiscium pleiostachyum) con $23.94 \mathrm{t}$ $\mathrm{CO}_{2} \mathrm{ha}^{-1}$, otras especies $68.36 \mathrm{t} \mathrm{CO}_{2} \mathrm{ha}^{-1}$. El total de carbono fijado para todas las especies de $257.56 \mathrm{t} \mathrm{CO}_{2}$ ha $^{-1}$ (Figura 10). Se puede notar que el sistema silvopastoril está fijando muy buena cantidad de dióxido de carbono, debido a que los árboles en su mayoría presentan buenos contenidos de carbono en su biomasa en diámetros mayores.

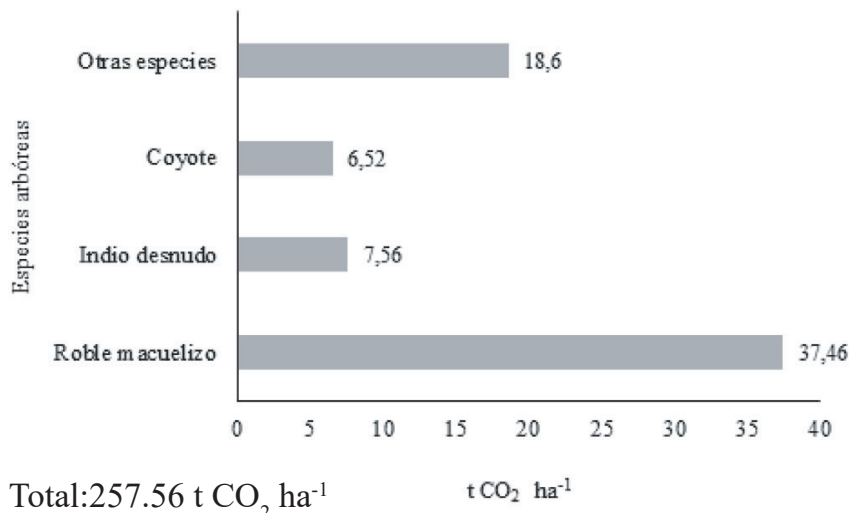

Figura 10. Carbono fijado $\mathrm{CO}_{2}$ por especie $\left(\mathrm{t} \mathrm{CO}_{2} \mathrm{ha}^{-1}\right)$ en siete fincas en los sistemas Silvopastoriles, Mulukukú, 2018.

Carbono almacenado total en sistemas silvopastoril. La especie que presenta la mayor cantidad de carbono almacenado es el Roble macuelizo (Tabebuia rosea) con 37.46 $\mathrm{C}$ ha $^{-1}$, en segundo lugar, indio desnudo (Bursera simarouba) con $7.56 \mathrm{C} \mathrm{ha}^{-1}$, y en tercer lugar el coyote (Platymiscium pleiostachyum) con $6.52 \mathrm{C} \mathrm{ha}^{-1}$, otras especies $18.6 \mathrm{C} \mathrm{ha}^{-1}$. El total de carbono almacenado para todas las especies de 70.18 $\mathrm{C} \mathrm{ha}{ }^{-1}$. Los resultados de contenido de carbono en sistema silvopastoril, es debido que la cobertura arbórea presenta una alta cantidad de árboles ha $^{-1}$ relacionado con las dimensiones en diámetro y altura, a pesar que se encontró una baja riqueza de especies en cada sitio evaluado.

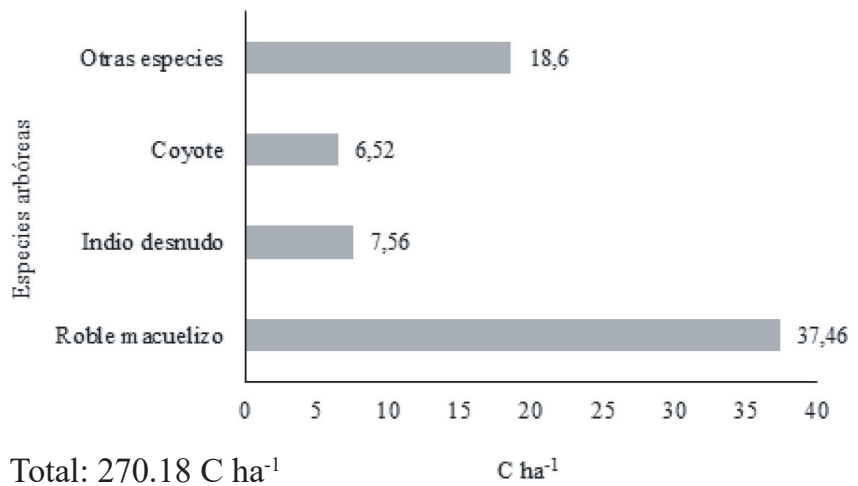

Figura 11. Carbono almacenado por especie $\left(\mathrm{C} \mathrm{ha}^{-1}\right)$ en siete fincas en los sistemas Silvopastoriles, Mulukukú, 2018.

\section{CONCLUSIONES}

El sistema bosque a través del componente arbóreo y de su alta densidad de árboles participa activamente en la fijación y almacenamiento de carbono, en el estudio fue notorio que en sistemas silvopastoriles los resultados fueron inferiores, debido a que el arbolado está en menor densidad, los resultados muestran que el bosque tiene mayor potencial en la producción de biomasa y contenidos de carbono debido a su alta densidad arbórea y riqueza florística que presenta este sistema.

El sistema bosque presentó mayor promedio de carbono fijado y almacenado con $157.12 \mathrm{t} \mathrm{ha}^{-1} \mathrm{y} 42.80 \mathrm{t} \mathrm{ha}^{-1}$, en comparación al sistema silvopastoril que presentó valores inferiores de $36.39 \mathrm{t} \mathrm{ha}^{-1}$ y $10.02 \mathrm{t} \mathrm{ha}^{-1}$, la densidad arbórea o el grosor del diámetro son características que inciden en la fijación y acumulación de carbono en la biomasa del arbolado.

En el sistema bosque la finca La Ceiba presentó los valores más altos en cuanto a contenido de carbono que la finca Regalo de Dios, mientras que en el sistema silvopastoril la finca Santa Emilia registró mayores resultados en comparación al resto de fincas.

El mayor promedio de biomasa y carbono almacenado lo presento el sistema bosque con $85.62 \mathrm{t} \mathrm{ha}^{-1}$ y $42.80 \mathrm{t} \mathrm{ha}^{-1}$, en comparación al sistema silvopastoril que presento valores inferiores de $20.05 \mathrm{t} \mathrm{ha}^{-1}$ y $10.02 \mathrm{t} \mathrm{ha}^{-1}$.

\section{RECOMENDACIONES}

Fomentar la diversidad florística en el sistema silvopastoril (especie forrajeras maderables y frutales) con el objetivo de mejorar el incremento en la producción de biomasa y fomentar un modelo diversificado de los servicios y atributos que proporcionan los árboles al ganado, así como a los propietarios de las fincas.

Identificar diferentes opciones de manejo para cada finca considerando las exigencias de crecimiento de las especies, la variabilidad climática y las prácticas de manejo.

Continuar con la evaluación de las parcelas permanentes de muestreo, las que permitan determinar la dinámica del carbono en la biomasa vegetal en el sistema bosque y silvopastoril en las fincas de este municipio.

Realizar manejo forestal del componente arbóreo, que debe ser vinculado con las condiciones biofísicas el que permita llegar a un equilibrio entre la productividad y la conservación de las fincas. 


\section{REFERENCIAS BIBLIOGRÁFICAS}

Brown, S. (1997). Estimating biomass and biomass change of tropical forests: a primer. (FAO Forestry Paper - 134). http://www.fao. org/3/w4095e/w4095e00.htm

Gasparri, I., Manghi, E., Montenegro, C., Parmuchi, G., Bono, J., y Strada, M. (2004). Estimación de volumen, biomasa y contenido de carbono de las regiones forestales argentinas. https://aulavirtual.agro.unlp.edu.ar

Instituto Nacional Forestal (2009). Resultados del Inventario Nacional Forestal: Nicaragua 2007-2008. http://www.cambioclimatico. ineter.gob.ni/bibliografia/Mitigacion $\% 20 \mathrm{del} \% 20$ cambio $\% 20$ climatico/Informe $\% 20$ Final $\% 20$ inventario $\% 20$ forestal.pdf

Instituto Nicaragüense de Estudios Territoriales. (2009). Consideraciones preliminares sobre la microlocalizacion de sitios para mataderos, industriales e industrias lacteas y harina. https://docplayer.es/amp/71661739-Consideraciones-preliminares-sobre-lamicrolocalizacion-de-sitios-para-mataderos-industriales-e-industrias-lacteas-y-harina.html

Jenkins, J., Ginzo, H., Ogle, S., Verchot, L., Handa, M., y Tsunekawa, A. (2006). Directrices del IPCC de 2006 para los inventarios nacionales de gases de efecto invernadero. https://www.ipcc-nggip.iges.or.jp/public/2006gl/spanish/pdf/4_Volume4/V4_08_Ch8_ Settlements.pdf

Mejía Bayas, M. M. (2013). Determinación del factor de forma de gmelina (Gmelina arbórea Roxb) en plantaciones de uno, dos y tres años de edad en la hacienda El Vergel cantón Valencia provincia de Los Ríos. Escuela Superior Politécnica de Chimborazo. http:// dspace.espoch.edu.ec/bitstream/123456789/2993/1/33T0121\%20.pdf

Ordóñez Jurado, H., Muñoz Guerrero, D., Ballesteros Possu, W., Cabrera Chamorro, G., y Mosquera González, J. (2007). Estimación de la biomasa aérea y captura de carbono en árboles dispersos en potreros con motilón silvestre (Freziera canescens) en el municipio de Pasto Nariño - Colombia. https://revistas.udenar.edu.co/index.php/rfacia/article/view/88/94

Organización de las Naciones Unidas para la Alimentación y la Agricultura. (1998). Directrices para la evaluación en los países tropicales y subtropicales. http://www.fao.org/3/ae218s/AE218S00.htm

Ramírez, D., Ordaz, J., Mora, J., Acosta, A., y Serna, B. (2010). Nicaragua efectos del cambio climático sobre la agricultura. https:// repositorio.cepal.org/bitstream/handle/11362/25925/1/lcmex1964.pdf

Rügnitz, M., Chacón, M., y Porro, R. (2009). Guia para la determinación de carbono en pequeñas propiedades rurales. https://www. academia.edu/3365683/Rugnitz_M._Chac\%C3\%B3n_M._Porro_R._2009._Guia_para_Determina\%C3\%A7\%C3\%A3o_de_ Carbono_em_Pequenas_Propriedades_Rurais._1._ed._--_Centro_Mundial_Agroflorestal_ICRAF_Cons\%C3\%B3rcio_Iniciativa_ Amaz $\%$ C $3 \% \bar{B} 4$ nica_IA_._2009_Bel\%C3\%A9m_Brasil._- 81 _p

Ugalde, L. (1981). Conceptos básicos de dasometría. http://repositorio.bibliotecaorton.catie.ac.cr/bitstream/handle/11554/886/ Conceptos_basicos_de_dasometria.pdf?sequence $=1 \&$ is Allowed $=y$

Walker, W., Baccini, A., Nepstad, M., Horning, N., Knight, D., Braun, E., y Bausch, A. (2011). Guía de campo para la estimación de biomasa y carbono vegetal. https://documents.pub/document/estimacion-de-biomasa-y-carbono-vegetalpdf.html 